\title{
Highly sensitive soft tactile sensors for an anthropomorphic robotic hand
}

\author{
Lorenzo Jamone, Lorenzo Natale, Giorgio Metta and Giulio Sandini
}

\begin{abstract}
The paper describes the design and realization of novel tactile sensors based on soft materials and magnetic sensing. In particular, the goal was to realize i) soft, ii) robust, iii) small and iv) low-cost sensors that can be easily fabricated and integrated on robotic devices that interact with the environment; we targeted a number of desired features, the most important being v) high sensitivity, vi) low hysteresis and vii) repeatability. The sensor consists of a silicone body in which a small magnet is immersed; an Hall-effect sensor placed below the silicone body measures the magnetic field generated by the magnet, which changes when the magnet is displaced due to an applied external pressure. Two different versions of the sensor have been manufactured, characterized and mounted on an anthropomorphic robotic hand; experiments in which the hand interacts with real world objects are reported.
\end{abstract}

Index Terms - Tactile sensors, soft materials, robotic hand, high sensitivity.

\section{INTRODUCTION}

Tactile sensing is fundamental in any application where contacts with the environment are expected, especially if such an environment is unstructured and cannot be fully determined and described, e.g. minimal invasive surgery (MIS), rehabilitation, virtual reality, telepresence, automation of small-medium enterprises (SMEs) and robotics [1], [2]. In particular, modern autonomous robots that are expected to coexist with humans, sharing their living and working environments, performing useful tasks while adapting to the surrounding space and reacting to unpredictable events, need tactile sensing for a number of reasons, ranging from safe operation (safe for the robot itself, for the humans, for the objects around) to behaviors that depend on human guidance [3]. Moreover, the sense of touch can dramatically improve the cognitive abilities of robots (e.g. object recognition and classification [4], autonomous self-calibration [5], motor learning [6]).

In order to cope with this increasingly pressing demand, over the past thirty years many tactile sensors have been proposed (see [7] for an extensive review, up to the year 2010). Even just during the last five years, a considerable number of solutions have been proposed, employing many different technologies: capacitive [8]-[11], optical [12], [13], piezoresistive [14][16] (see [17] for a recent review), piezoelectric [18]-[20], ultrasonic [21], magnetic [22]-[24], nanoparticles [25], carbon

L. Jamone is with the Instituto de Sistemas e Robótica, Instituto Superior Técnico, Lisbon, Portugal, e-mail: ljamone at isr.ist.utl.pt.

L. Natale and G. Metta are with the iCub Facility, Istituto Italiano di Tecnologia, Genoa, Italy, email: lorenzo.natale,giorgio.metta at iit.it

G. Sandini is with the Department of Robotics, Brain and Cognitive Sciences, Istituto Italiano di Tecnologia, Genoa, Italy, giulio.sandini at iit.it Manuscript received XXX; revised XXX.

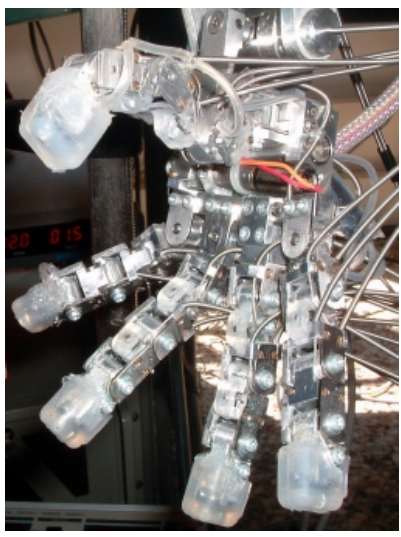

Fig. 1. Anthropomorphic robotic hand equipped with 12 tactile sensors.

nanotubes [26], [27], conductive liquids [28]-[30], conductive polymers [31] and tunnel effect [32].

Unfortunately, only a few of these technologies have been tested in actual robots, and therefore it is not easy to evaluate to what extent the data extracted from these sensors is useful for robotic applications. In particular, anthropomorphic robotic hands present additional constraints that have to be met in terms of size, weight and complexity (i.e. number of wires and connections) of the sensors: the hand dexterity should not be affected. Example of sensors that have been successfully integrated in anthropomorphic robotic hands and used in real world experiments are in [8], [24].

A few companies have also started to produce tactile sensors for robotic applications, for example the FlexiForce from Tekscan [33], the 3D Force Sensor from OptoForce [34], the QTC sensors from Peratech [35] and the BioTac fingertip from Syntouch [36]. However, the price of these devices is still relatively high, and the sensor performance is not always sufficient for the desired task.

In this paper we describe the design, development and realization of a novel tactile sensor that is particularly suited for sensorizing an anthropomorphic robotic hand. The sensor main body is made of a silicone elastomer, which offers a good balance between softness and robustness; moreover, we show that mechanical hysteresis is very limited. The transduction technology is magnetic; together with the physical properties of the silicone, this solution provides high sensitivity of the measurement. The components of the sensor are small, cheap and easily retrievable; we describe in detail how the sensors can be fabricated, mounted (in our case, on a multifingered robot hand) and tested. Additionally, we report robotic 
experiments in which the sensors have been successfully used to detect contacts in different tasks.

Initial prototypes of the sensors have been presented in a previous paper [37]; here we report their full characterization, that has been performed only recently, and we describe their fabrication in more detail.

The rest of the paper is organized as follows. In Section II we outline the structure of the sensor, and in Section III we present the characterization of the output. Then, in Section IV we introduce the humanoid robot that we equipped with the developed sensors, and in Section V we report real world experiments. Finally, in Section VI we draw our conclusions and sketch the future work.

\section{SENSOR STRUCTURE}

The sensor consists of three main parts: a soft body, a magnet and a Hall-effect sensor. The basic structure and working principle is depicted in Fig. 2. A small magnet is immersed in the soft body, which lies over the Hall-effect sensor. The Hall-effect sensor measures the magnetic field generated by the magnet: the intensity of this magnetic field around the sensor is proportional to the distance between the magnet and the sensor. When pressure is applied on the external side of the soft body the magnet is displaced toward the sensor, and the intensity of the magnetic field measured by the sensor increases. The opposite happens when the pressure is released.

The idea of using Hall-effect sensors and magnets to measure a tactile response was originally proposed in [38] and [39], where only preliminary prototypes were presented, and then not investigated anymore until recently [23], [24], [37], [40]. The work in [23] proposes a design which is very similar to the one we present; however, the sensor is still at a prototype stage, it is not integrated on a robot hand or other similar devices, and no dynamic characterization is reported, therefore if is not easy to evaluate the quality of the measurements, especially in terms of the sensor hysteresis. The work described in [24], [40] is instead more mature, and it has been successfully applied to real robotic scenarios; however, even if many simulation analysis are presented, also this work lacks a complete characterization of the real sensor (no dynamic, no repeatability, no hysteresis). Moreover, the design they propose (with four Hall Effect sensors) imposes constraints on the minimum size of the whole system, and the minumum detectable force seems to be considerably higher then our sensor (by a factor of ten).

Since our goal was to install the sensors on an anthropomorphic robotic hand, size constraints were imposing the use of small components. As Hall-effect sensor we use a Miniature Ratiometric Linear Hall Effect Sensor (Honeywell SS490 Series, see right side of Fig. 3). As permanent magnet we choose a cylindrical one with $2 \mathrm{~mm}$ diameter and a height of $1.5 \mathrm{~mm}$ (see left side of Fig. 3, near to a 1 euro cent coin); after some initial testing, we choose a magnet of grade N35 (the maximum energy product $B \cdot H_{\max }$ is 35 megagauss oersteds). The characteristic curve of the Hall-effect sensor (as a function of the vertical distance from the magnet)

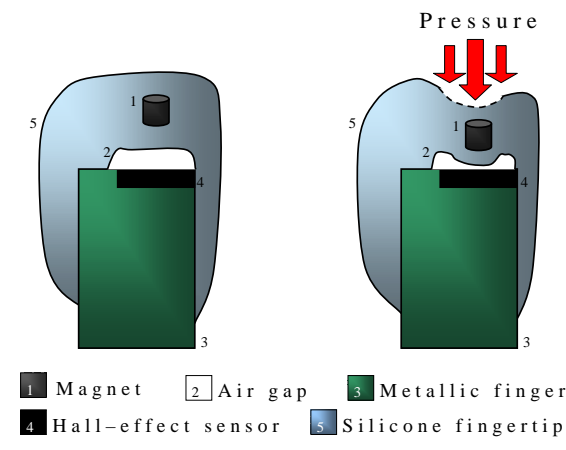

Fig. 2. Structure of the sensor and sensing principle. Note the presence of an air gap between the silicone shell and the Hall Effect sensor, that increases the sensitivity of the system.

has been obtained by measuring the analog output of the sensor at different distances from the magnet (see Fig. 4); the sensor was powered with 5 volts, providing an output between 2.5 and 5 volts. As for the silicone, after having tried a few alternatives we decided for the SYLGARD 186 from Dow Corning [41]. It is a two-part elastomer: two viscous components (base and curing agent) are mixed together at room temperature, and then cured at increasing temperatures to finally obtain the solid-state silicone part (more details will be given later in this Section). While the product datasheet suggests a 1:10 ratio between the two components, we were testing different ratios in order to obtain the desired elasticity and robustness of the solid-state silicone, and we eventually used a ratio of 1:8 for the final versions of our sensors.

Noticeably, in order to improve the sensitivity of the sensor, we decided to insert a small air gap between the bottom of the silicone shell and the Hall-effect sensor (as sketched in Fig. 2), instead of directly placing the silicone in contact with the Hall-effect sensor.

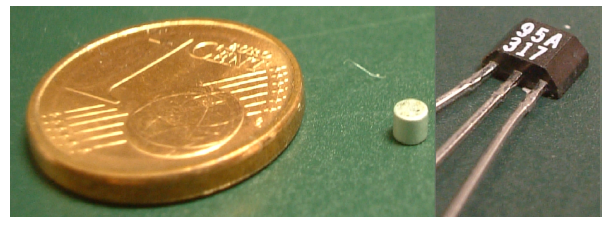

Fig. 3. Hall-effect sensor and magnet, near to a 1 euro cent coin (for size comparison).

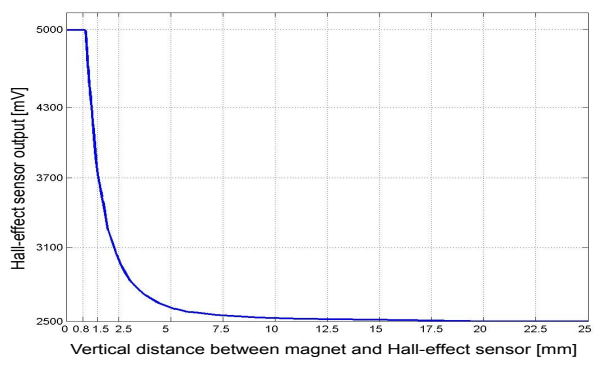

Fig. 4. Hall-effect sensor characteristic curve. 
After investigating a number of prototypes, the final outcome of the design procedure are two different models of the sensor, that are meant to be mounted on different parts of the robot hand: the fingertip model and the phalangeal model (see left and right images in Fig. 5).

The fingertip models are mounted on the hand fingertips, and each of them presents two sensing elements (i.e. two magnets and two Hall-effect sensors): one on the tip of the finger, the other on the finger pad. The design of the geometric and elastic properties of the silicone shell allowed to minimize the cross-talk between the two sensing elements; indeed, cross-talk is absent for stimuli (i.e. normal force applied) up to $3 N$. The silicone shell has no electric connection with the fingertip, and it can be easily installed and removed just by pushing and pulling (only adding a bit of sealing silicone after it is installed): this is an important aspect for the maintainance of the sensor, since the part that directly contacts the environment can get damaged more easily.

The phalangeal models are mounted on the internal sides of the fingers, and each of them has one sensing element. This model is smaller in size and weight, and easier to fabricate; it has been designed to be extremely sensitive, at the cost of allowing measurements in a smaller range with respect to the fingertip model. Also in this model, an air gap separates the magnet and the Hall Effect sensor.

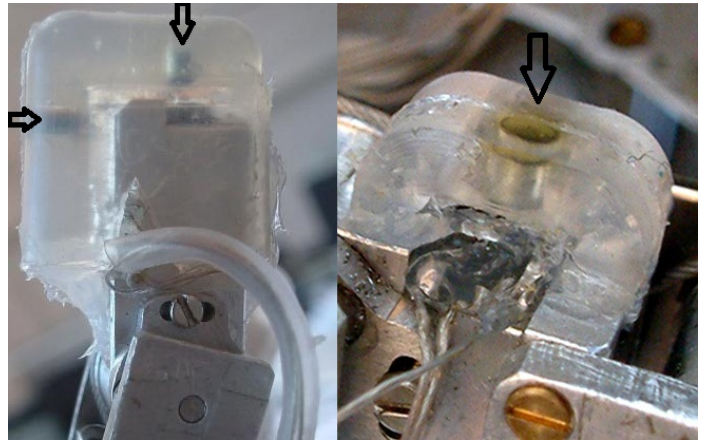

Fig. 5. Final versions of the sensors: on the left, the fingertip model, on the right, the phalangeal model.

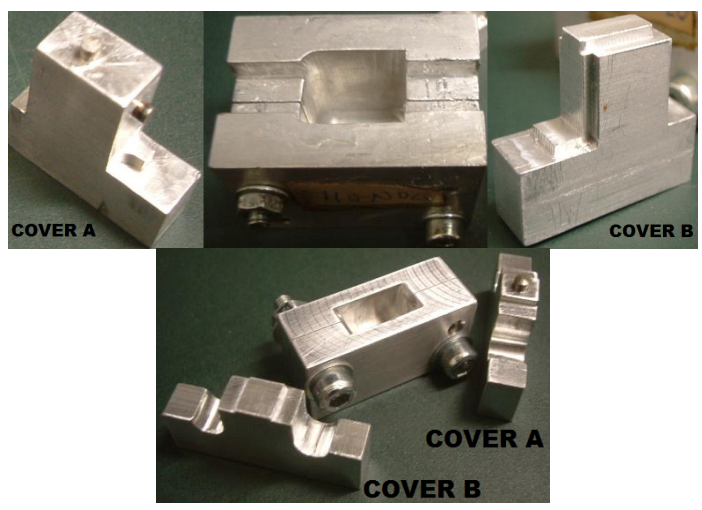

Fig. 6. Molds used to fabricate the silicone shells: top image, for the fingertip model, bottom image, for the phalangeal model.

We designed two different metallic molds to fabricate the silicone shells needed for the sensors (see Fig. 6); the fabrication procedure is the same for both sensor models. Two different covers for each mold can be seen in the figures: cover A, with little cylindrical bulges to produce suitable holes for the magnets, and cover B, with rabbets on some edges to create the already mentioned air gaps. Even if in our case the molds are metallic, and have been realized with a CNC (Computer Numerically Controlled) milling machine, they can also be realized in plastic with (nowadays widely available) $3 \mathrm{D}$ printing technology.

Molds are first filled with some viscous silicone (mixture of the two parts, base and curing agent), covered with cover A and left curing under progressive heating (as suggested in the product datasheet): first 24 hours at room temperature and then, in the oven, 4 hours at $65^{\circ} \mathrm{C}, 1$ hour at $100^{\circ} \mathrm{C}$ and 20 minutes at $150^{\circ} \mathrm{C}$. It should be noted that curing at increasing temperature it is not strictly needed, as it simply reduces the curing time: the whole procedure can be done at room temperature as well. Afterward, magnets are inserted, some more silicone is poured, and molds are covered with cover B and left curing again. When ready, silicone parts are fixed on the fingers with a sealing silicone (for securing them in place), on top of the Hall effect sensors previously glued in the desired positions.

\section{ChaRACTERIZATION OF THE SENSORS}

In this section we report the measurements that we performed to characterize the output of the different models of the sensor. Data was acquired using a measurement setup that includes i) a Cartesian robot (IAI Table Top TT robot), ii) a force/torque (F/T) sensor (ATI nano 17) mounted on the robot end-effector, and iii) a data acquisition board (NI DAQ USB6008) to read the Hall-effect sensors signals. A cylindrical probe, with a flat end surface of about $10 \mathrm{~mm}^{2}$ area, was attached to the robot end-effector to stimulate the sensor. The setup was used in previous work [8]. The Hall-effect sensors are powered with 5 volts power supply, and produce output signals that do not need to be further amplified; an offset (i.e. zero of the sensor) is computed in the beginning of the measurements, when no contact is present, and subtracted from the sensor signal, so that the sensor reads zero when there is no contact. Fig. 7 shows the setup during one of the test sessions; the sensors have been tested while mounted on the robot finger.

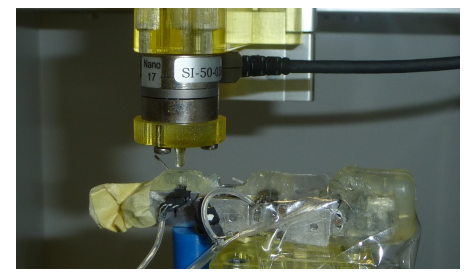

Fig. 7. Measurement setup used to characterize the sensors output: a Cartesian robot whose probe (i.e. end-effector) is instrumented with a F/T sensor. In the bottom of the image the robot finger equipped with the tactile sensors is shown. 


\section{A. Fingertip model}

We report here the characterization of the fingertip model. All the plots in this section are related to one of the sensing elements of the fingertip model (i.e. the one placed on the internal side of the fingerpad); the plots related to the other sensing element (i.e. the one placed on the top of the fingertip) are very similar and therefore not shown here.

Fig. 8 shows the characteristic curve of the fingertip model, describing the relationship between the normal force applied on the silicone shell and the voltage output of the Hall-effect sensor. Static data points have been obtained during 20 sets of measurements; for each data point we computed mean and standard deviation. The different forces were applied for about 10 seconds each, with an interval of about 10 seconds between each measurement. The blue solid line shows the interpolation of the measurements mean values, while the vertical bars indicate the standard deviations. The low values of standard deviation are a proof of the repeatability of the measurements. For the fingertip model, we target to measure loads between $0 N$ and about $3 N$, with the output saturating at $4 N$; indeed, this is the range we expect to deal with when using our robot hand for manipulation, considering the actuation limits of the robot fingers, and it is also the typical range of both normal and tangential forces experienced by humans during daily manipulation [42]. The interpolated data points (blue solid line) are compared to a reference linear interpolation (thick green dashed line) made of two distinct segments, one between $0 N$ and $2 N$, and another one between $2 N$ and $3 N$. A different linear interpolation (thin red dashed line), composed of a single interpolant segment (from $0 N$ to $3 N$ ), is also shown for reference. The sensor output saturates for loads higher than $4 N$, when the magnet is at the minimum distance from the Hall-effect sensor; higher loads further compress the silicone layer above the magnet, which absorbs the applied force limiting potential damages to the rest of the structure (i.e. compliant behaviour).

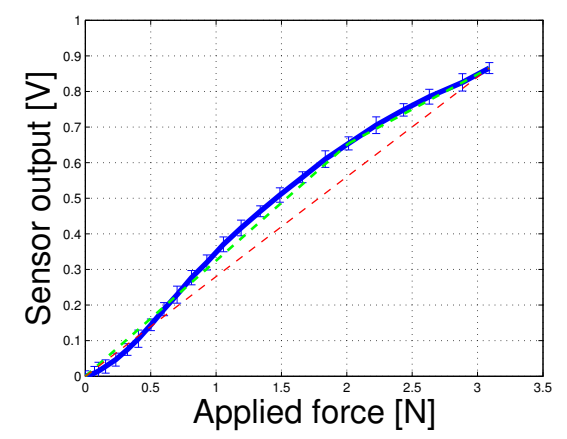

Fig. 8. Characteristic curve of the fingertip model. The blue solid line shows the interpolation of the test points, that are located in correspondence of the small vertical bars; the bars indicate the standard deviation with respect to the average, over a set of 20 measurements. Two linear interpolations (green and red dashed lines) are shown for reference.

The minimum detectable force is $0.05 N$, with a voltage output of $0.01 \mathrm{~V}$ (after filtering the measurement, the maximum noise is $0.002 \mathrm{~V}$ ); given that the force is applied on an area of about $10 \mathrm{~mm}^{2}$, this results in a pressure of about $5 k P a$.
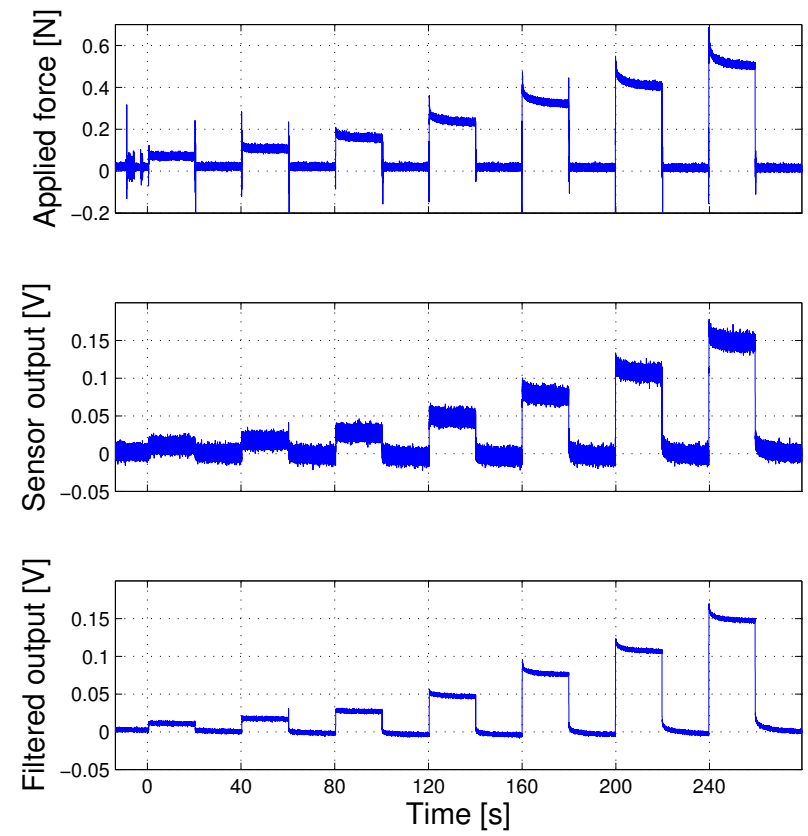

Fig. 9. Consecutive stimulations of the fingertip model with different normal forces. Top image: intensity of the applied force. Middle image: raw sensor output. Bottom image: filtered sensor output.
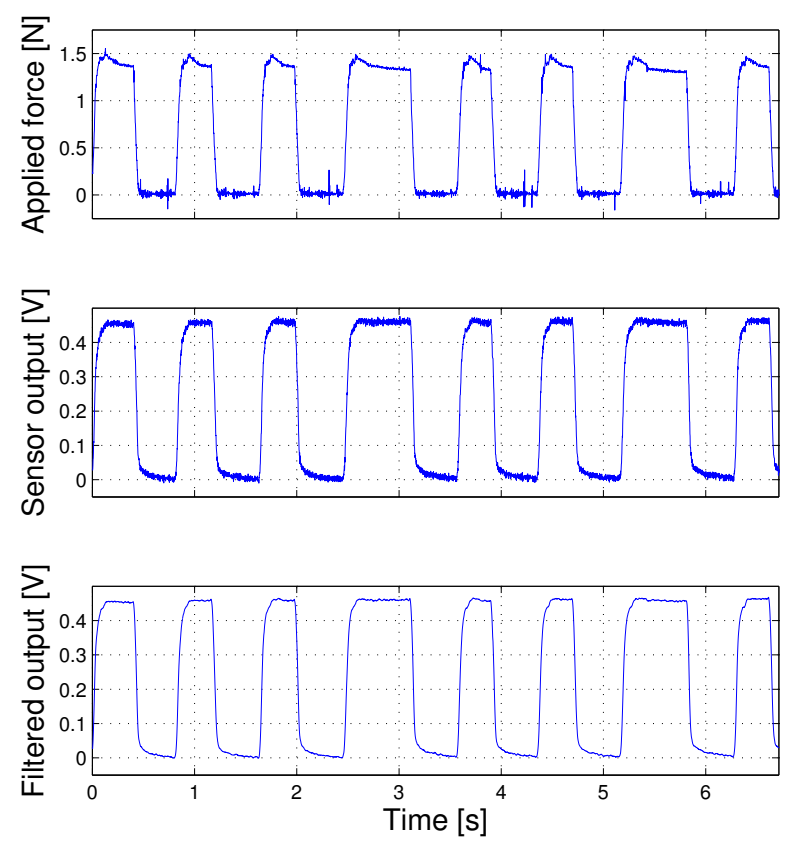

Fig. 10. High-rate consecutive stimulations of the fingertip model with the same normal force. Top image: intensity of the applied force. Middle image: raw sensor output. Bottom image: filtered sensor output. 
The plots in Fig. 9 and Fig. 10 depict the dynamic behavior of the sensor. Two different experiments have been performed. In the first one (Fig. 9) the sensor was stimulated with forces of increasing intensity; each stimulation was lasting about 20 seconds, with an interval of about 20 seconds between each stimulation. In the second experiment (Fig. 10) the sensor was stimulated at a much faster rate, with the same applied force $(1.4 \mathrm{~N}$ at steady state); each stimulation was lasting about 0.5 seconds, with an interval of about 0.5 seconds between each stimulation (note that the stimulation is sometimes a bit longer, due to the control limitations of the Cartesian robot at high speeds). In both figures, the top image plots the normal force measured by the F/T sensor (i.e. the force applied on the sensor), the middle image plots the raw output of the Hall-effect sensor, and the bottom image plots the filtered output. We used a Savitzky-Golay filter [43] to smooth the sensor output. It can be noticed from this set of plots that the mechanical hysteresis is very low: the sensor reads the applied forces correctly, and quickly returns to a null output as soon as the applied force is removed. Moreover, the sensor response is almost instantaneous (less than $20 \mathrm{~ms}$ ). This dynamic tests also show an interesting phenomenon that is related to the soft properties of the silicone shell: the force measured by the F/T sensor has an initial transient, before reaching a quasisteady level. This happens because, soon after the contact, part of the impact force is dissipated into the elastic body of the silicone. This passive compliance shown by our sensors make them particularly suited for operating in unknown and unstructured environments, where unexpected impacts may damage the sensor and/or the external environment if no compliant behavior is present.

\section{B. Phalangeal model}

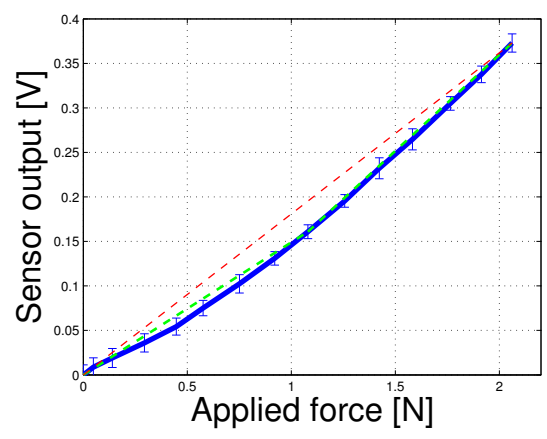

Fig. 11. Characteristic curve of the phalangeal model. The blue solid line shows the interpolation of the test points, that are located in correspondence of the small vertical bars; the bars indicate the standard deviation with respect to the average, over a set of 20 measurements. Two linear interpolations (green and red dashed lines) are shown for reference.

We report here the characterization of the phalangeal model. The meaning of the plots (Fig. 11, Fig. 12 and Fig. 13) is the same as for the fingertip model. For the phalangeal model we target a higher sensitivity, at the cost of a slightly smaller range of measurements, in this case between $0 N$ and about $2 N$. The sensor output saturates for loads higher than $3 N$. The minimum detectable force is $0.01 \mathrm{~N}$, with a voltage output of
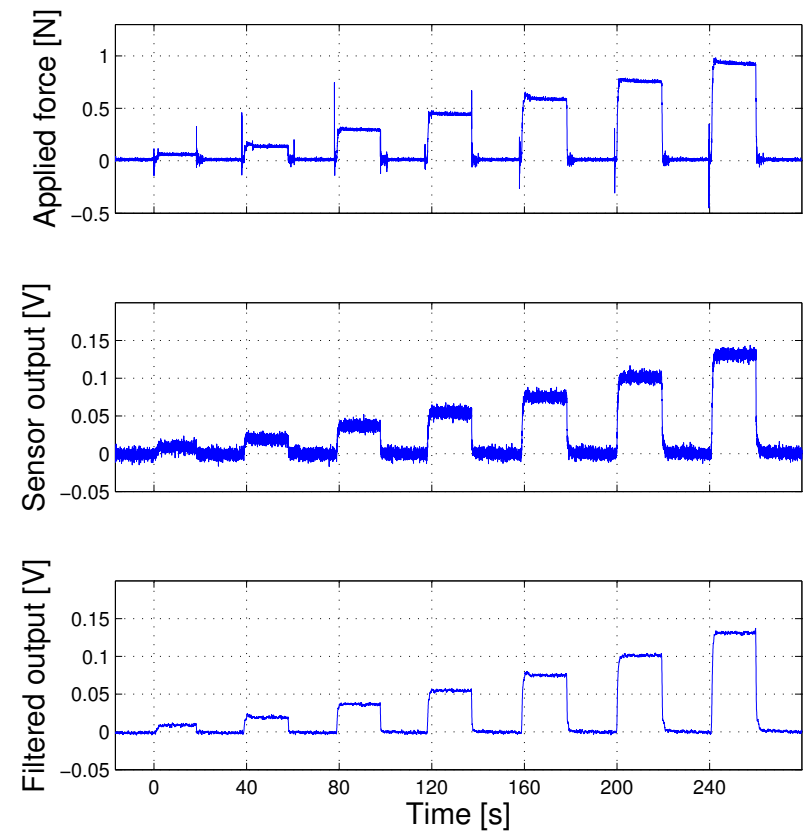

Fig. 12. Consecutive stimulations of the phalangeal model with different normal forces. Top image: intensity of the applied force. Middle image: raw sensor output. Bottom image: filtered sensor output.
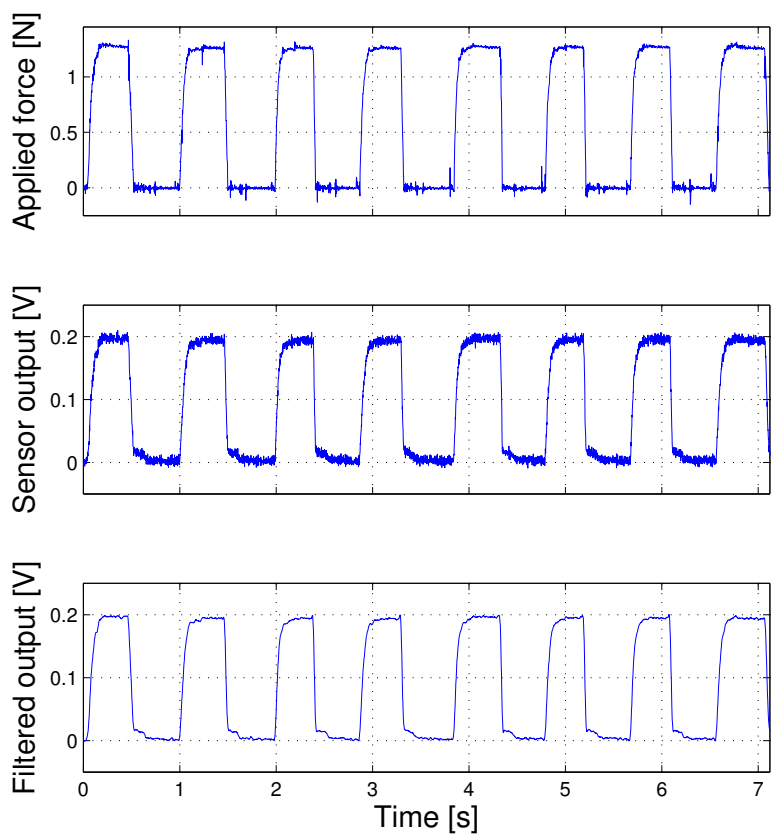

Fig. 13. High-rate consecutive stimulations of the phalangeal model with the same normal force. Top image: intensity of the applied force. Middle image: raw sensor output. Bottom image: filtered sensor output. 
$0.002 \mathrm{~V}$ (after filtering the measurement, the maximum noise is $0.001 \mathrm{~V}$ ); given that the force is applied on an area of about $10 \mathrm{~mm}^{2}$, this results in a pressure of about $1 \mathrm{kPa}$.

In general, the results show that the same considerations we made for the fingertip model (i.e. low mechanical hysteresis, fast response, compliant behavior) are applicable to the phalangeal model.

\section{THE HUMANOID PLATFORM}

The sensors described in this paper have been designed and developed in order to sensorize the robotic hand (see Fig. 1) of James, a 22 DOFs upper body humanoid. The robot has been designed taking in consideration a scenario of object manipulation in real unstructured environments, and therefore a great deal of attention was put into the design of an highly anthropomorphic hand, tactile and force sensing, and an intrinsically compliant actuation system. Specifically, the compliance is obtained by using plastic belts and stainlesssteel tendons to transmit torques from motors to joints; moreover, springs have been employed in critical locations, both along the tendons (to increase their elasticity) and as structural parts, as for example in the neck (see [44]-[46] for a detailed description of the neck structure, sensorization and control). The hand has five fingers. Each of them has three joints (flexion/extension of the distal, middle and proximal phalanxes). Two additional degrees of freedom are represented by the thumb opposition and by the coordinated abduction/adduction of four fingers (index, middle, ring, and little finger). Therefore, the hand has a total of 17 joints, actuated by motors located in the arm (the torque transmission is obtained through stainless-steel tendons). A more detailed description of the robot can be found in [37].

\section{A. Installation of the sensors}

The robot hand has been equipped with five fingertip sensors, one for each finger, and seven phalangeal sensors, two on the thumb, ring finger and middle finger, and one on the index finger (see Fig. 1); in total, 17 tactile sensing elements are present on the hand. This was the maximum number of sensing elements allowed, due to the limitations in the available ADC channels in the acquisition board. The 17 Hall-effect sensors are connected to an acquisition board mounted on the hand back, that is interfaced to a CAN communication bus that routes all the sensor and motor signals of the robot. The acquisition board is based on a PIC18F448 microcontroller, an ADC (analog-to-digital converter) and multiplexer, and a connector that receives the signals and provides the 5 Volt DC power supply to the sensors. The same board receives also the signals of the proprioceptive sensors of the hand (i.e. measurements of the joint angles of the fingers), that are also realized using Hall-effect sensors; in total, 40 wires are connected to the board through the connector. To connect all these sensors in such a little space, with a reasonable resiliency (required because of the constant interaction of the hand with the external environment), we have chosen a very thin stainless-steel cable, coated in Teflon, with a 0.23 $\mathrm{mm}$ external diameter. Moreover, in order to further increase system robustness, cables are grouped into silicone catheters along their route between different sensors and toward the acquisition board (as it can be seen in Fig. 1, for example along the thumb).

\section{EXPERIMENTS}

We briefly report here two previously published experiments that were performed using the sensors presented in this paper, mounted on the robot hand. In the first experiment (Section V-A) we show how the high sensitivity of the sensors can be exploited to control the interaction of the robot hand with the environment. Then, in the second experiment (Section V-B) we demonstrate how the sensors can provide important information about grasped object, in particular about properties that cannot be extracted through vision (e.g. softness).

\section{A. Withdrawal reflex of the robot wrist}

We consider here a reaching movement during which the hand accidentally contacts an obstacle that has to be avoided: to achieve this, the robot bends the wrist trying to cancel the tactile stimulation (i.e. to eliminate the contact), realizing a sort of withdrawal reflex.

The robot hand contacts a static obstacle during an arm movement performed at the average speed of about $0.5 \mathrm{~m} / \mathrm{s}$, generating contact forces up to $2 N$ on the different fingers (see Fig. 14). The top image in Fig. 14 depicts the wrist pitch joint trajectory during this motion, elicited by the tactile sensors stimulations shown in the bottom image of Fig. 14. It can be noticed that four tactile elements are stimulated in sequence: the first is the phalangeal-sensor of the index finger, the second and third are the phalangeal-sensors of the middle finger and the last one is the fingertip-sensor of the middle finger. This indicates that the hand slithered on the obstacle during the motion. During the interaction the wrist is controlled with a velocity proportional to the intensity of the (overall) tactile stimulation. When there is no contact, wrist position is restored to zero with a proportional controller. More details can be found in [47].

\section{B. Classification of grasped objects}

We present here one result from [37] that shows the importance of tactile sensors for classifying grasped objects. We trained two Self Organizing Maps (SOMs), with different sensory data: one with proprioceptive data (i.e. positions of the hand joints) and the other with both proprioceptive and tactile data. The images in Fig. 15 show a classification test done on two objects that have similar shape but different softness: a plastic bottle and a woolen scarf wrapped in a cylindrical shape. Interestingly, while the SOM in the top image (the one without tactile data) clusters the two objects in the same class, the bottom SOM is able to correctly separate the two objects.

\section{CONCLUSIONS AND FUTURE WORK}

We reported the design, realization and experimental characterization of a novel tactile sensor based on magnetic sensing, 


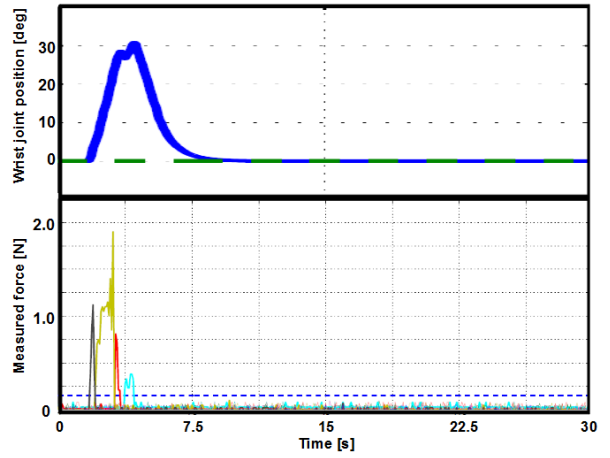

Fig. 14. Tactile obstacle avoidance motion. Top image: trajectory of the pitch joint of the wrist. Bottom image: readings from the hand tactile sensors.

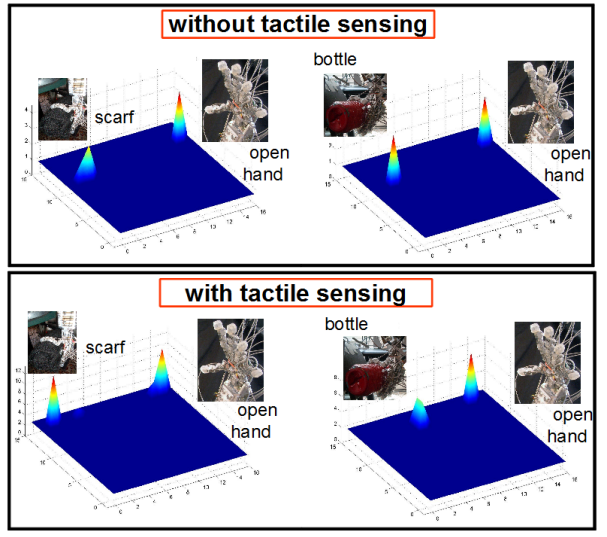

Fig. 15. Classification of grasped objects. Two objects with similar shape but different softness are tested: scarf and bottle. Top image: without tactile sensing (objects are considered in the same class). Bottom image: with tactile sensing (objects are correctly separated)

particularly suited for robotic applications. To build the main structure of the sensor we use a silicone elastomer that offers an optimized balance between robustness and softness. We realized two versions of the sensor to sensorize a multifingered robotic hand. The components employed are cheap and easily retrievable, and the fabrication of the sensors does not require any special machine. Therefore, it is easy for researchers to realize their own sensors based on the proposed idea, and following the guidelines we reported: we believe that this is an important contribution to the scientific community, especially for roboticists.

The realized sensors measure the normal component of the applied force, with high sensitivity, low hysteresis and good repeatability. In particular, the minimum sensed force is $0.05 \mathrm{~N}$ for the fingertip model, and $0.01 \mathrm{~N}$ for the phalangeal model; to the best of our knowledge, these values correspond to the state of the art for tactile sensors that have been successfully integrated in robotic hands. This has been achieved through careful design choices, both in terms of mechanical properties of the silicone elastomer (that can be optimized by modifying the ratio between base and curing agents) and in terms of design of the whole structure (e.g. inclusion of the air gap between the bottom part of the silicone layer and the Halleffect sensor).

We report real world experiments showing two possible robotic applications: tactile-based obstacle avoidance and haptic classification of grasped objects. This is a further proof that the sensors are mechanically robust and can be used in real tasks. In the versions proposed here, the sensor measures only the normal component of the applied force (1-D measurement). However, a full 3-D measure of the applied force can be obtained by replacing the 1-D Hall Effect sensor that we currently employ with a 3-D Hall Effect sensor (an idea investigated in [23]). Moreover, the proposed versions have either one or two sensing elements each: futher engineering of the sensor structure may lead to versions with multiple sensing elements. This has been initially suggested in [39], even if no details were provided. These are the two main lines we plan for the future development of this technology.

\section{ACKNOWLEDGMENT}

This work was partially funded by the EU Projects POETICON++ [FP7-ICT-288382] and LIMOMAN [PIEFGA-2013-628315].

\section{REFERENCES}

[1] M. Lee, "Tactile sensing: New directions, new challenges," Int. J. Robot. Res., vol. 19, no. 7, pp. 636-643, 2000.

[2] J. Tegin and J. Wikander, "Tactile sensing in intelligent robotic manipulation a review," Ind. Robot, vol. 32, no. 1, pp. 64-70, 2005.

[3] B. Argall and A. Billard, "A survey of tactile human-robot interactions," Robot. Auton. Syst., vol. 58, pp. 1159-1176, 2010.

[4] M. Schopfer, "Tactile perception of cognitive robots," Ph.D. dissertation, Universitt Bielefeld, 2011.

[5] A. Roncone, M. Hoffmann, U. Pattacini, and G. Metta, "Automatic kinematic chain calibration using artificial skin: self-touch in the icub humanoid robot," in ICRA. IEEE, 2014.

[6] K. Kronander and A. Billard, "Learning compliant manipulation through kinesthetic and tactile human-robot interaction," IEEE T. Haptics, vol. 7, no. 3, pp. 367-380, 2014

[7] R. S. Dahiya, G. Metta, M. Valle, and G. Sandini, "Tactile sensingfrom humans to humanoids," IEEE T. Robot., vol. 26, no. 1, pp. 1-20, 2010.

[8] A. Schmitz, P. Maiolino, M. Maggiali, L. Natale, G. Cannata, and G. Metta, "Methods and technologies for the implementation of largescale robot tactile sensors," IEEE T. Robot., vol. 27, no. 3, pp. 389-400, 2011.

[9] C. Hong, L. Chu, W. Lai, A.-S. Chiang, and W. Fang, "Implementation of a new capacitive touch sensor using the nanoporous anodic aluminum oxide (np-aao) structure," IEEE Sens. J., vol. 11, no. 12, pp. 3409-3416, 2011.

[10] Y.-C. Liu, C.-M. Sun, L.-Y. Lin, M.-H. Tsai, and W. Fang, "Development of a cmos-based capacitive tactile sensor with adjustable sensing range and sensitivity using polymer fill-in," J. Microelectromech. S., vol. 20, no. 1, pp. 119-127, 2011.

[11] H. Muhammad, C. Recchiuto, C. Oddo, L. Beccai, C. Anthony, M. Adams, M. Carrozza, and M. C. L. Ward, "A capacitive tactile sensor array for surface texture discrimination," Microelectron. Eng., vol. 88, pp. 1811-1813, 2011.

[12] A. Massaro, F. Spano, A. Lay-Ekuakille, P. Cazzato, R. Cingolani, and A. Athanassiou, "Design and characterization of a nanocomposite pressure sensor implemented in a tactile robotic system," IEEE T. Instrum. Meas., vol. 60, no. 8, pp. 2967-2975, 2011.

[13] B. Ali, M. A. Ayub, and H. Yussof, "Characteristics of a new optical tactile sensor for interactive robot fingers," Int. J. Soc. Robot., vol. 4, pp. 85-91, 2012.

[14] Y. Hu, R. B. Katragadda, H. Tu, Q. Zheng, Y. Li, and Y. Xu, "Bioinspired 3-d tactile sensor for minimally invasive surgery," J. Microelectromech. S., vol. 19, no. 6, pp. 1400-1408, 2010.

[15] A. Kimoto and Y. Matsue, "A new multifunctional tactile sensor for detection of material hardness," IEEE T. Instrum. Meas., vol. 60, no. 4, pp. 1334-1339, 2010.

[16] K. Yamashita, Y. Yang, T. Nishimoto, K. Furukawa, and M. Noda, "Piezoelectric vibratory-cantilever force sensors and axial sensitivity analysis for individual triaxial tactile sensing," IEEE Sens. J., vol. 13, no. 3, pp. 1074-1080, 2013. 
[17] S. Stassi, V. Cauda, G. Canavese, and C. F. Pirri, "Flexible tactile sensing based on piezoresistive composites: A review," Sensors, vol. 14, pp 5296-5332, 2014

[18] S. K. Hwang and H. Y. Hwang, "Development of a tactile sensing system using piezoelectric robot skin materials," Smart Mater. Struct., vol. 22, no. 4, pp. $055004-055012,2013$.

[19] L. Seminara, L. Pinna, M. Valle, L. Basiric, A. Loi, P. Cosseddu, A. Bonfiglio, A. Ascia, M. Biso, A. Ansaldo, D. Ricci, and G. Metta, "Piezoelectric polymer transducer arrays for flexible tactile sensors," IEEE Sens. J., vol. 13, no. 10, pp. 4022-4029, 2013.

[20] H.-J. Tseng, W.-C. Tian, and W.-J. Wu, "Flexible pzt thin film tactile sensor for biomedical monitoring," Sensors, vol. 13, pp. 5478-5492, 2013.

[21] K. Kim, S. Zhang, J. Tian, P. Han, and X. Jiang, "Face-shear mode ultrasonic tactile sensor array," in IUS, 2012.

[22] M. Goka, H. Nakamoto, and S. Takenawa, "A magnetic type tactile sensor by gmr elements and inductors," in IROS. IEEE, 2010.

[23] C. Ledermann, S. Wirges, D. Oertel, M. Mende, and H. Woern, "Tactile sensor on a magnetic basis using novel $3 \mathrm{~d}$ hall sensor - first prototypes and results," in INES. IEEE, 2013.

[24] S. Youssefian, N. Rahbar, and E. Torres-Jara, "Contact behavior of soft spherical tactile sensors," IEEE Sens. J., vol. 14, no. 5, pp. 1435-1442, 2014.

[25] D. Alvares, L. Wieczorek, B. Raguse, F. Ladouceur, and N. H. Lovell, "An evaluation study of nanoparticle films as biomimetic tactile sensors," Sensors Actuat. A-Phys., vol. 186, pp. 148-153, 2012.

[26] C. F. Hu, W. S. Su, and W. Fang, "Development of patterned carbon nanotubes on a $3 \mathrm{~d}$ polymer substrate for the flexible tactile sensor application," J. Micromech. Microeng., vol. 21, no. 11, pp. 115012 $1115012-12,2011$.

[27] O. Yilmazoglu, A. Popp, D. Pavlidis, J. J. Schneider, D. Garth, F. Schuttler, and G. Battenberg, "Vertically aligned multiwalled carbon nanotubes for pressure, tactile and vibration sensing," Nanotechnology, vol. 23, no. 8, pp. 85 501-85 508, 2012.

[28] Y. L. Park, B. R. Chen, and R. J. Wood, "Design and fabrication of soft artificial skin using embedded microchannels and liquid conductors," IEEE Sens. J., vol. 12, pp. 2711-2718, 2012.

[29] D. M. Vogt, Y. L. Park, and R. J. Wood, "Design and characterization of a soft multi-axis force sensor using embedded microfluidic channels," IEEE Sens. J., vol. 13, pp. 4056-4064, 2012.

[30] F. L. H. III, R. K. Kramer, Q. Wan, R. D. Howe, and R. J. Wood, "Soft tactile sensor arrays for force feedback in micromanipulation," IEEE Sens. J., vol. 14, no. 5, pp. 1443-1452, 2014.

[31] T. Zhang, H. Liu, L. Jiang, S. Fan, and J. Yang, "Development of a flexible 3-d tactile sensor system for anthropomorphic artificial hand,' IEEE Sens. J., vol. 13, no. 2, pp. 510-518, 2013.

[32] Y. Amarasinghe, A. Kulasekera, and T. Priyadarshana, "Quantum tunneling composite (qtc) based tactile sensor array for dynamic pressure distribution measurement," in ICST, 2013.

[33] "Tekscan - FlexiForce," http://www.tekscan.com/flexiforce.html, accessed: 2014-09-24

[34] "Optoforce - 3D Force Sensor," http://optoforce.com/3dsensor/, accessed: 2014-09-24.

[35] "Peratech - QTC Touch Processing Unit," http://www.peratech.com/qtctouch-processing-unit.html, accessed: 2014-09-24.

[36] "Syntouch - BioTac," http://www.syntouchllc.com/Products/BioTac/, accessed: 2014-09-24

[37] L. Jamone, F. Nori, G. Metta, and G. Sandini, "James: A humanoid robot acting over an unstructured world," in Humanoids. IEEE, 2006

[38] J. Clark, "A magnetic field based compliance matching sensor for high resolution, high compliance tactile sensing," in ICRA. IEEE, 1988.

[39] W. Nowlin, "Experimental results on bayesian algorithms for interpreting compliant tactile sensing data," in ICRA. IEEE, 1991.

[40] E. Torres-Jara, I. Vasilescu, and R. Coral, "A soft touch: Compliant tactile sensors for sensitive manipulation," MITCSAIL, Tech. Rep. MITCSAIL-TR-2006-014, 2006.

[41] "Dow corning - SYLGARD 186 silicone elastomer kit," http://www.dowcorning.com/applications/search/default.aspx?R=118EN, accessed: 2014-09-24.

[42] H. E. Wheat, L. M. Salo, and A. W. Goodwin, "Human ability to scale and discriminate forces typical of those occurring during grasp and manipulation," J. Neurosci., vol. 24(13), pp. 3394-3401, 2004.

[43] A. Savitzky and M. Golay, "Smoothing and differentiation of data by simplified least squares procedures," Anal. Chem., vol. 36, pp. 1627-39, 1964.

[44] F. Nori, L. Jamone, G. Metta, and G. Sandini, "Accurate control of a human-like tendon driven neck," in Humanoids. IEEE, 2007.
[45] M. Fumagalli, L. Jamone, G. Metta, L. Natale, F. Nori, A. Parmiggiani, M. Randazzo, and G. Sandini, "A force sensor for the control of a human-like tendon driven neck," in Humanoids. IEEE, 2009.

[46] L. Jamone, L. Natale, M. Fumagalli, F. Nori, G. Metta, and G. Sandini, "Machine-learning based control of a human-like tendon driven neck," in ICRA. IEEE, 2010.

[47] L. Jamone, M. Fumagall, L. Natale, F. Nori, G. Metta, and G. Sandini, "Control of physical interaction through tactile and force sensing during visually guided reaching," in $M S C$. IEEE, 2014.

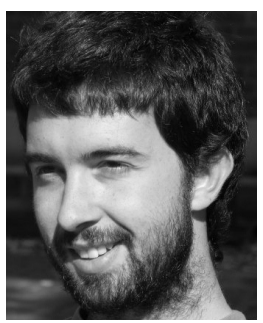

Lorenzo Jamone received his MS in Computer Engineering from the University of Genova in 2006 (with honors), and his $\mathrm{PhD}$ in Humanoid Technologies from the University of Genova and the IIT in 2010. He was Associate Researcher at the Takanishi Laboratory in Waseda University from 2010 to 2012, and since January 2013 he is an Associate Researcher at VisLab (Instituto Superior Técnico, Lisbon, Portugal). His research interests include cognitive humanoid robots, motor learning and control, force and tactile sensing.

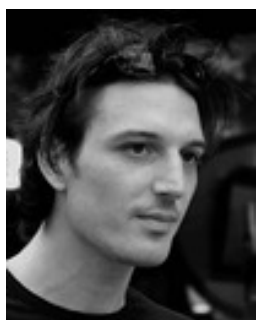

Lorenzo Natale received his degree in Electronic Engineering (with honors) in 2000 and $\mathrm{PhD}$ in Robotics in 2004 from the University of Genova. Between 2005 and 2006 he was Postdoctoral researcher at the MIT Computer Science and Artificial Intelligence Laboratory, in the Humanoid Robotics Group. He is now team leader in the iCub Facility at IIT. His research focuses on developmental robotics, sensorimotor learning and perception in artificial and biological systems.

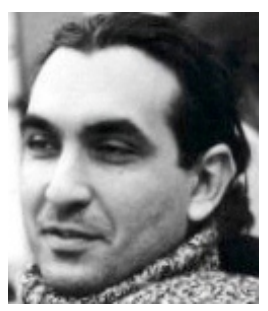

Giorgio Metta is Director of the iCub Facility at IIT and assistant professor at the University of Genova. He holds a MS with honors (in 1994) and $\mathrm{PhD}$ (in 2000) in electronic engineering both from the University of Genova. From 2001 to 2002 he was Postdoctoral associate at the MIT AI-Lab. He is assistant professor at the University of Genova since 2005 and with IIT since 2006. He is author of more than 300 publications, mainly in the fields of biologically motivated and humanoid robotics.

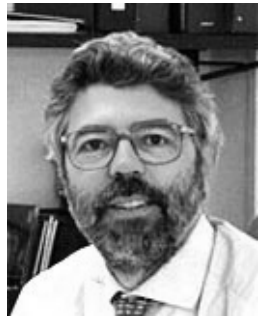

Giulio Sandini is Director of the department of Robotics, Brain and Cognitive Sciences at IIT (since 2006) and full professor of bioengineering (since 1984) at the University of Genova, where he founded the LIRA-Lab in 1990. He graduated in Electronic Engineering at the University of Genova in 1976 and he was research fellow and assistant professor at the Scuola Normale Superiore in Pisa until 1984. He is author of more than 500 publications. His research activities are in the fields of Biological and Artificial Vision, Computational Neuroscience and Robotics. 